\title{
Non-convex flux functions and compound shock waves in sediment beds
}

\author{
Gert Bartholomeeusen ${ }^{1}$, Hans De Sterck ${ }^{2}$, and Gilliane Sills ${ }^{3}$ \\ 1 University of Oxford, Department of Engineering Science, Parks Road, Oxford \\ OX1 3PJ, UK gert.bartholomeeusen@eng.ox.ac.uk \\ 2 University of Colorado at Boulder, Department of Applied Mathematics, \\ Colorado CO-80309-0526 desterck@colorado.edu \\ 3 University of Oxford, Department of Engineering Science, Parks Road, Oxford \\ OX1 3PJ, UK gilliane.sills@eng.ox.ac.uk
}

Summary. Sediment layers deposited under water undergo a deformation that for low soil concentrations can be described by a scalar nonlinear hyperbolic conservation law. The associated flux function is non-convex and compound shock waves arise, which are shocks followed or preceded by a rarefaction with the shock speed equal to the wave speed at the point of attachment. The paper describes an experimental study of compound shock waves in sediment beds and the numerical modelling of the sedimentation process using an experimentally obtained flux function for kaolinite soil. The work also fits in a broader investigation of the transition parameter between the physical processes of soil sedimentation and consolidation, as the data obtained allows identification of this parameter.

\section{Introduction}

In soil sedimentation processes soil particles settle in a fluid as a group, driven by a flux function which only depends on the particle concentration. The mathematical formulation is simply given by the continuity equation which is classified as a hyperbolic partial differential equation:

$$
\frac{\partial n}{\partial t}+\frac{\partial f(n)}{\partial x}=0
$$

where $n$ is the porosity, defined as the volume of voids divided by the total volume. Given an initial uniform porosity in a settling experiment, different types of shock waves will propagate through the sediment depending on the precise form of the flux function, $f(n)$. For soil suspensions very little is known about the form of the flux function or about the convexity or non-convexity of the function. However, detailed experimental proof of discontinuities propagating in sediments dates from the early 1980's [3], and has later been confirmed by others [1]. This paper presents soil sedimentation experiments, in which shocks are tracked by carefully monitoring surface settlement and X-ray density or porosity profiles, to obtain a suitable flux function for the kaolinite soil used. The flux function is validated by using it 
in a numerical solution of equation 1, which is compared with experimental results.

The group settling of particles dependent only on the local concentration, often also called hindered settling, defines the sedimentation process, and the experiments performed in this study are focussed on the range of porosities where this physical mechanism is dominant. When suspensions become more dilute, drag forces around the larger particles will move the smaller ones up, leading to the process of differential settling. Next to an upper bound in porosity for the sedimentation process, there is a lower bound for denser suspensions, where the particle interactions provide a supporting framework which influences the settling process. This process is known to geotechnical engineers as large strain consolidation [7]. Neither the upper or lower bound processes are considered in this paper.

Kynch [8] was the first to formulate the theory of sedimentation, which was later mainly applied in chemical engineering problems, such as fluidisation. Often artificial particles were used, eg glass beads and rubber balls, but some work was also performed on soil sediments. Michaels \& Bolger [10] showed that the theory of sedimentation applies to non-equisized particles and flocculated suspensions. In geotechnical engineering problems the volume reduction of a suspension bed by sedimentation is typically in the order of $50 \%$, and therefore a good understanding is of great importance for design and management purposes. In spite of this in practice soil sedimentation is most often treated as an instantaneous collapse of the suspension bed.

\section{Wave Structures}

The soil sedimentation process starts from a uniformly mixed soil-water suspension, and subsequent temporal evolution is characterised by an increasing clear water layer appearing at the surface and a more compact soil bed building-up from the base. The experiments presented in this paper are carried out in settling columns with an internal diameter of $101 \mathrm{~mm}$. The soil used is industrially exploited kaolinite, which has a variety of applications, eg in the paper industry. It is chosen here for its very fine grain size: 90 percent of the particles, $d_{90}$, have a diameter smaller than 8 micron and 10 percent, $d_{10}$, is smaller than 0.7 micron. Therefore, it is most likely that the region of porosities at which differential settling occurs will be fairly narrow and the sedimentation region can also be found not too far from water $(n=1)$.

Figure 1 shows the results of a settling experiment in which the evolution of the porosity profiles is monitored by measurement of X-rays passing through a settling column containing a kaolinite suspension. The method provides an accuracy of measurement of porosity of \pm 0.0013 and a spatial resolution of $\pm 1 \mathrm{~mm}[3,12]$. At the 29 min profile a thin, more compact layer has formed at the base, which is divided from the initial porosity by a discontinuity. At the top another discontinuity separates the initial porosity 
from that of water. With the passage of time the two discontinuities move towards each other as shock waves, as seen in the 98 and 177 min profiles. Three distinct parts can be identified: a lower porosity bed, the initial porosity layer and a water layer. Eventually the two shock fronts meet each other, and further compaction occurs. This process of sedimentation in a settling column experiment, described by equation 1, can be modelled as a double Riemann problem with two wave fronts moving towards each other.

Shocks can occur in various ways depending on the flux function $f(n)$ and the position of the initial porosity on it. If porosity discontinuity, such as the water-suspension interface or the suspension-bed interface, is plotted in $(x, t)$ space, shock waves can be classified in terms of the characteristic lines that intersect the shock. A regular shock wave occurs when the characteristic lines (with slope $f^{\prime}(n)$ ) enter the discontinuity from both sides. Then the speed of the shock is determined by the Rankine Hugoniot Jump Condition:

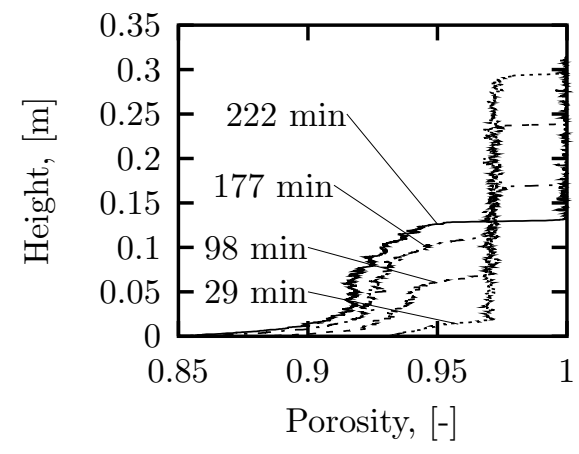

Fig. 1. Porosity profiles monitored by means of X-rays. $\left(h_{i}=0.315 \mathrm{~m}\right.$ and $\left.n_{i}=0.972\right)$.

$$
s=\frac{f\left(n_{l}\right)-f\left(n_{r}\right)}{n_{l}-n_{r}} .
$$

Equivalently if, with $n_{l}$ and $n_{r}$ the porosities on the left and right of the shock, respectively, the entropy condition [11]

$$
\frac{f(n)-f\left(n_{l}\right)}{n-n_{l}} \geqslant s \geqslant \frac{f(n)-f\left(n_{r}\right)}{n-n_{r}} ;
$$

is satisfied for all $n$ between $n_{l}$ and $n_{r}$, a regular shock occurs. When condition 3 is not fulfilled, the relevant solution is a rarefaction wave or a compound shock wave. When the local wave speed diverges away from the shock on the left and right side, a rarefaction wave is obtained. For a compound shock wave, characteristic lines go into the shock on one side, while on the other side the characteristic line or local wave speed is parallel to the shock.

In order to illustrate these three different types of shock waves, simple flux functions are assumed. For the regular shock wave, a convex flux function $f(n)=n(1-n) / 2$ is taken. Expressions 2 and 3 applied to the sedimentation problem will, for any initial porosity, result in two regular shock waves, down- and upwards, as the characteristic lines go into the shock, see figure 2(a). Upon increasing the order by one, the now non-convex flux function, $f(n)=n^{2}(1-n) / 2$, contains an inflection point. The downward moving shock remains regular. However, from the base a rarefaction (figure 2(b)) or 
a compound shock wave (figure 2(c)) travels upwards. If the initial porosity, $n_{i}$, is smaller than or equal to the porosity at the inflection point, $n_{\mathrm{I}}$, a rarefaction will occur. A compound shock wave propagates when $n_{i}>n_{\mathrm{I}}$. Note that all the porosities from the characteristic line of the top rarefaction down to $n=0$ are included in the rarefaction. Different forms of compound shock waves can occur if the number of inflection points increases. As long as there is no inflection point between $n=1$ and the porosity that corresponds to the maximum flux, a regular shock wave is always obtained for the sediment-water interface.

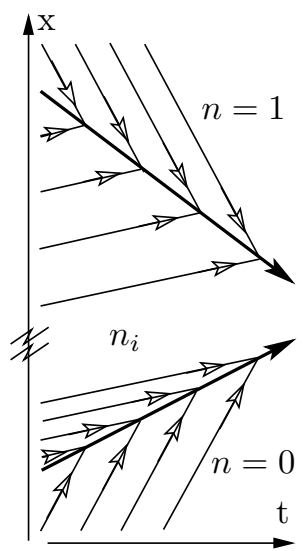

(a)

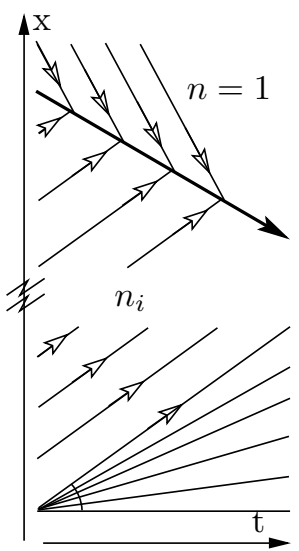

(b)

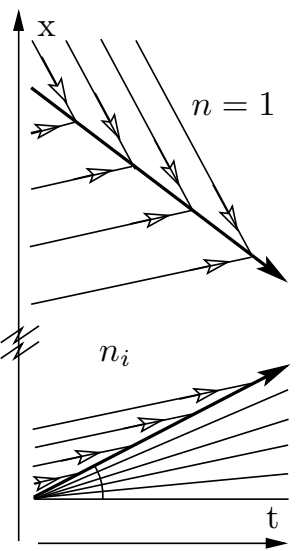

(c)

Fig. 2. Illustration of possible wave structures in sedimentation. (a) Regular shock wave down- and upwards. (b) Regular shock wave down and rarefaction wave up. (c) Regular shock wave down and compound shock wave up.

It is well-known that compound shocks arise in systems described by hyperbolic conservation laws with non-convex flux functions, i.e, for which wave speeds do not vary in a monotonic manner with relevant changes of the state variables. Many physical systems that can be described by hyperbolic conservation laws have flux functions with quadratic nonlinearities that are convex. For example, the scalar Burgers equation and the system of Euler equations for gas dynamics are convex such that compound shocks do not occur. More complex flux functions may be obtained when real gases are modelled, which may allow for the occurrence of compound shocks. An early example of a non-convex scalar flux function is given by the Buckley-Leveret equation which models two-phase flows that pertain to oil recovery problems [9]. This equation exhibits a cubic flux function. The system of magnetohy- 


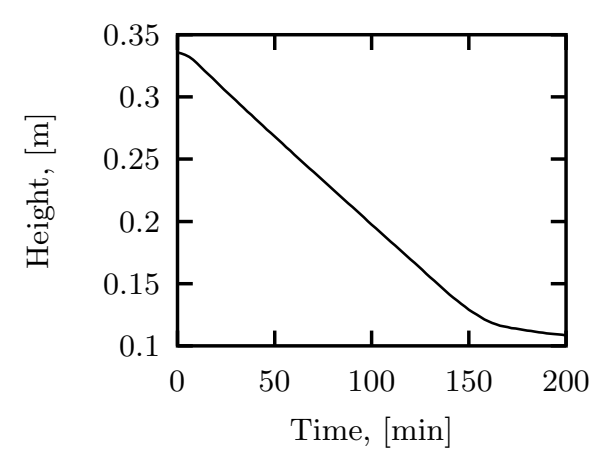

(a)

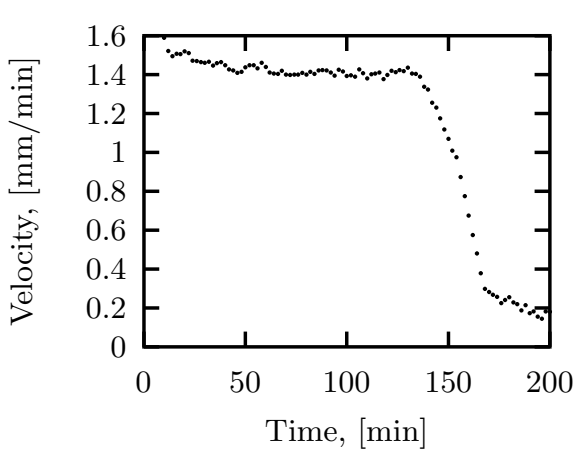

(b)

Fig. 3. Surface settlement (a) and surface velocity (b) - Kaolin suspension $h_{i}=0.335 \mathrm{~m}$ or $n_{i}=0.979$.

drodynamics equations has two non-convex modes [4] and exhibits compound shocks, which have been found in numerical simulations in one, two and three spatial dimensions $[4,5,6]$.

\section{Experimental Observations}

The flux function of kaolinite suspensions can be determined from experimental observations by applying the Rankine-Hugoniot Jump condition, equation 2. For initial porosities between that corresponding to the maximum flux and unity (water), digital imaging has been used to locate the sediment-water interface with an accuracy better than $0.6 \mathrm{~mm}$. The sediment surface settlement was monitored in thirteen such experiments, covering an initial porosity range from 0.94 to 0.98 . Figure 3(a) shows an example of the surface settlement, and it can be clearly seen that the sediment first settles at a constant rate and then reduces to a much slower settling rate. The speed of the surface sediment is depicted in figure 3(b), which makes this behaviour even more apparent. Around $140 \mathrm{~min}$ the constant speed reduces from $1.4 \frac{\mathrm{mm}}{\mathrm{min}}$ to 0.2 $\frac{\mathrm{mm}}{\min }$. At the time the surface velocity drops, the associated regular surface shock wave meets either the first characteristic line of a rarefaction or the shock of a compound shock wave, see figures 2(b) and 2(c). During the time of constant settling velocity, the average is taken as the shock speed, $s$, and with the porosities above and below the surface shock known, expression 2 can be solved for the flux of the initial porosity as the flux of water $(n=1)$ is zero. For the experiments for which the compound shock wave (propagating up from the base of the column) has been tracked by means of X-ray density 


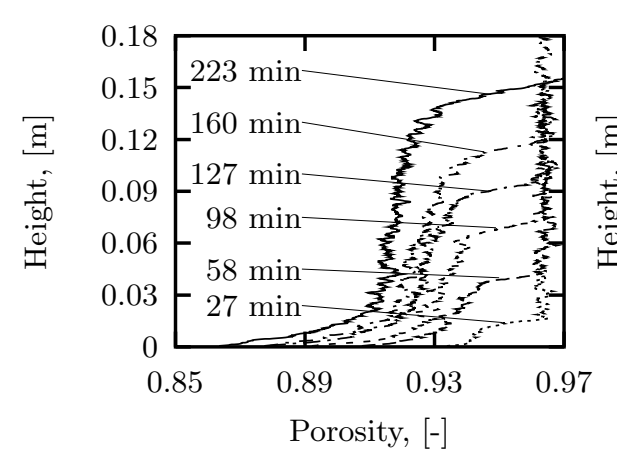

(a)

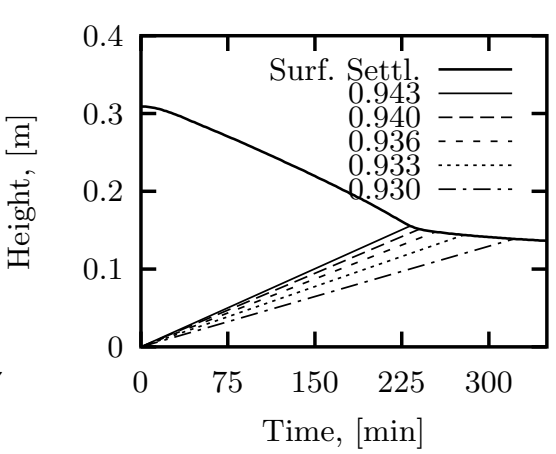

(b)

Fig. 4. X-ray shock track experiment - Kaolin suspension $h_{i}=0.309 \mathrm{~m}$ and $n_{i}=$ 0.964. (a) Porosity profiles, (b) Surface settlement and characteristic lines.

measurements, lines of constant porosity or characteristic lines can be constructed. The Rankine-Hugoniot Jump condition applies locally when such characteristic lines enter the surface shock, and flux can be calculated from equation 2. Figure 4(a) focuses on the upward travelling porosity shock of a settling column experiment. The behaviour is very similar to the porosity profile presented in figure 1 with compound shocks clearly visible. Characteristic lines, which are obtained by linear regression of points of constant porosity, are shown in figure 4(b) combined with the surface settlement.

The flux data obtained by the methods described above is depicted in figure 5. The thirteen surface settlement experiments have given thirteen flux points, while three shock track experiments using the X-ray system together resulted in eighteen points. Within the porosity range of $\sim 0.92$ to $\sim 0.95$ the shock track experiments define the left flank of the flux function consistently. The surface track data and the shock track data overlap in the region of $\mathrm{n}=0.94$ to 0.96 . Between this maximum and water $(n=1)$ a steady decrease in the flux is observed. The sedimentation experiments performed show that the flux function of kaolin is highly peaked. In order to perform numerical predictions of sedimentation experiments of kaolin suspensions a fitted function needs to be found. The basic condition for the function is that the flux needs to be zero for pure solids and water, respectively $n=0$ and $n=1$. Preferably the function should contain only one inflection point to avoid very complex wave structures [9]. By splitting the flux function up in three parts, simple power law expressions can be used:

$$
n=0 \rightarrow n=n_{\mathrm{I}}: \frac{f_{1}(n)}{f_{1}\left(n_{\mathrm{I}}\right)}=\left(\frac{n}{n_{\mathrm{I}}}\right)^{b}
$$




$$
\begin{gathered}
n=n_{\mathrm{I}} \rightarrow n=n_{\mathrm{M}}: \frac{f_{2}\left(n_{\mathrm{M}}\right)-f_{2}(n)}{f_{2}\left(n_{\mathrm{M}}\right)-f_{2}\left(n_{\mathrm{I}}\right)}=\left(\frac{n_{\mathrm{M}}-n}{n_{\mathrm{M}}-n_{\mathrm{I}}}\right)^{b^{\prime}} ; \\
n=n_{\mathrm{M}} \rightarrow n=1: \frac{f_{3}\left(n_{\mathrm{M}}\right)-f_{3}(n)}{\left.f_{3}\left(n_{\mathrm{M}}\right)-0\right)}=\left(\frac{n_{\mathrm{M}}-n}{1-n_{\mathrm{M}}}\right)^{b^{\prime \prime}} ;
\end{gathered}
$$

where $b, b^{\prime}$ and $b^{\prime \prime}$ are determined by calibration and fulfill the condition for continuous differentiability:

$$
\begin{gathered}
\left(\frac{d f_{1}}{d n}\right)_{n=n_{I}}=\left(\frac{d f_{2}}{d n}\right)_{n=n_{I}} ; \\
\left(\frac{d f_{2}}{d n}\right)_{n=n_{M}}=\left(\frac{d f_{3}}{d n}\right)_{n=n_{M}}=0 .
\end{gathered}
$$
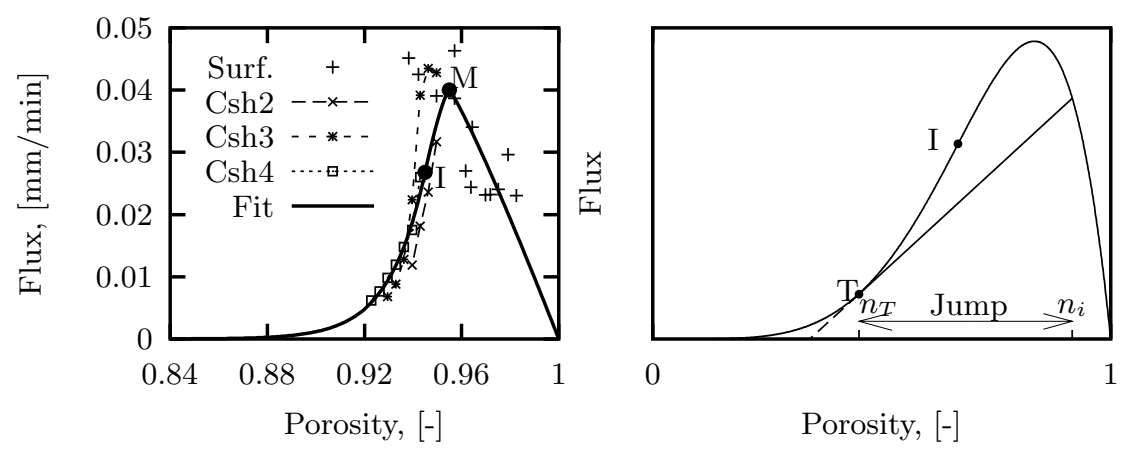

Fig. 5. Experimental Flux Data.

Fig. 6. Conceptual illustration of use of flux function and tangent lines.

The inflection point I and the maximum $\mathrm{M}$ are partly chosen by fitting functions $f_{1}(n)$ and $f_{3}(n)$ to the data and partly on an arbitrary basis. Having determined the inflection point I, compound shock waves will only occur for initial porosities larger than $n_{\mathrm{I}}$. The magnitude of the porosity jump can easily be determined by drawing a tangent line from the initial point $\left(n_{\mathrm{i}}, f\left(n_{\mathrm{i}}\right)\right)$ to the flux function and the corresponding difference in porosity equals the step magnitude (Jump) as illustrated in figure 6 . The speed of the shock attached to the rarefaction is determined as the slope of the tangent line from $n_{i}$ to the inside of the flux curve [9].

\section{Numerical Simulation Results}

The double Riemann problem described by equation 1 is numerically approximated by a conservative second order accurate slope limited scheme [9]. The 
conservation property of the numerical scheme results in correctly calculated wave speeds.

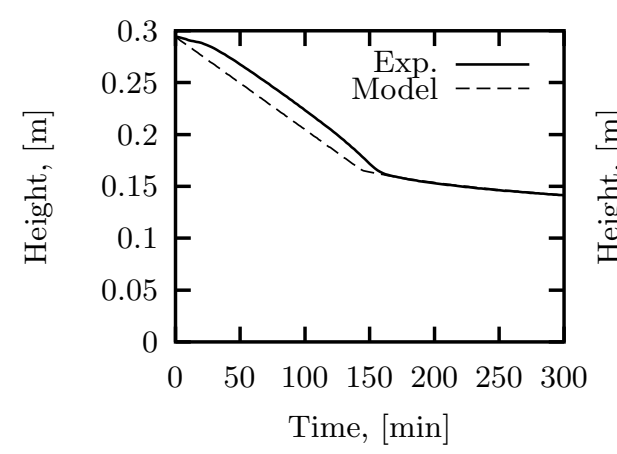

(a)

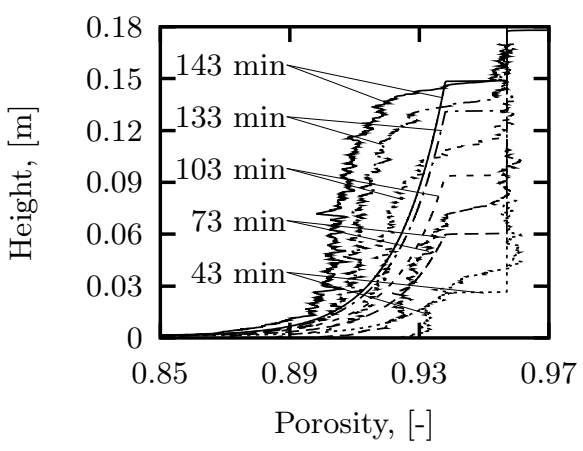

(b)

Fig. 7. Comparison - Kaolin suspension $h_{i}=0.295 \mathrm{~m}$ and $n_{i}=0.957$. (a) Surface settlement, (b) Porosity profiles with compound shocks composed of a shock with an attached rarefaction.

Figure $7(\mathrm{a})$ shows the comparison between simulation and a kaolinite settling column experiment with $h_{i}=0.295 \mathrm{~m}$ and $n_{i}=0.957$ using the experimentally obtained flux function. The model has clearly predicted the same constant settling rate before the slow down, but the experiment experiences a time-lag due to the turbulent energy of the mixing process, which cannot be predicted by the model. At the point the first characteristic meets the surface shock the settling slows down and the time-lag disappears and model and experiment are in accordance. The best way to evaluate the validity and quality of the sedimentation model is by comparing it to the upward travelling porosity shock. In order to make a realistic comparison of the porosity profiles, the time-lag of the experiment is shifted back to correspond with the model prediction. Figure 7(b) compares the experimental porosity profiles to the numerically simulated. At 43 and 73 minutes realistic approximations of the porosity shock moving upwards and the porosity profiles underneath the shock are obtained. At the base the model has a porosity that equals zero, though in the experimental profiles this is far greater. This feature becomes more apparent for the later profiles where the porosity decreases gradually. The predicted magnitude of the shock is good, while the tracking in time is relatively good and almost exact in the profiles of 133 and 143 minutes. The shapes of the experimental porosity profiles from the base up to the shock after 73 minutes are concavely shaped from the base upwards, but turn gradually over to a convex shape towards the shock. The model predicted profiles 
all have a concave shape from the base to the shock, with a significant amount of material contained at the base. Consolidation is a dissipative process and is thus not modelled by the dissipation-free hyperbolic equation 1 . It is in this dissimilarity in predicted and experimental porosity profiles, that the neglected diffusive particle interaction and consolidation process in equation 1 and in the theory of sedimentation becomes most apparent. The theory of soil consolidation [7] is built-upon the deformation by particle interaction only, and concave-convex porosity profiles can be predicted [2], though this model does not handle the experimentally observed compound shock waves.

\section{Conclusions}

It has been shown that by using the Rankine-Hugoniot Jump Condition, equation 2, an experimental flux function can be derived from the experiments performed. Three similar power law expressions, which together differentiate continuously and define one inflection point and a maximum, give a simple but accurate representation of the experimental data. The region of porosities where compound shock waves occur, is situated between $n_{\mathrm{I}}$ and 1 .

Numerical predictions of the surface settlement provide a good simulation of the constant settling rate and subsequent reduction in speed, despite the initially slower start of the experiment. The upward-shock tracking performance of the model is satisfactory and the size of the jump is adequate. Initially the soil bed porosity under the shock is also predicted well, but as the bed grows a differently shaped form is obtained due to the influence of inter-particle forces.

Overall it can be concluded that compound shock waves typify sedimentation and exist for kaolin suspensions only in a narrow region of initial porosities. It is clear from figure 7(b) that the dissipative effect of consolidation manifests itself in the whole rarefaction part of the compound shock. The question at which porosity consolidation first occurs for a given material has been investigated in the literature but has not been answered satisfactorily. The experiments and simulations reported in this paper suggest strongly that consolidation occurs in the whole rarefaction part of the compound shock. We thus conclude that, for a given material and a given experiment, the highest porosity for which consolidation occurs is the porosity at the characteristic line that connects the rarefaction to the compound shock (the tangent point in figure 6). This highest porosity does thus not only depend on the material, but also on the porosity above the compound shock, which is the uniform initial porosity in our sedimentation experiments. When varying this initial porosity in the experiments, the absolutely highest porosity for which consolidation can occur is the porosity corresponding to the inflection point $n_{\mathrm{I}}$ in figure 6 . This identifies clearly for which porosity the transition between sedimentation and consolidation occurs. 


\section{Acknowledgements}

Financial support from Dredging International and the Engineering and Physical Sciences Research Council is gratefully acknowledged. Prof Guy Houlsby of the Oxford University Engineering Department is thanked for many useful suggestions.

\section{References}

[1] Alves, M.-C. 1992. Compartamento de sedimentação e adensamento de uma lama vermelha. Ph.D. thesis, Pontificia Universidade Católica do Rio de Janeiro.

[2] Bartholomeeusen, G., Sills, G.C., Znidarčić, D., Van Kesteren, W., Merckelbach, L.M., Pyke, Robert, III, W.D. Carrier, Lin, H., Penumadu, D., Masala, S., \& Chan, D. accepted for publication. Sidere: Prediction of large strain consolidation. Géotechnique.

[3] Been, K., \& Sills, G.C. 1981. Self-weight consolidation of soft soils. Géotechnique, 31, 519-535.

[4] Brio, M., \& Wu, C.C. 1988. An upwind scheme for the equations of ideal magnetohydrodynamics. Journal of computational physics, 75, 400422.

[5] De Sterck, H., Low, B.C., \& Poedts, S. 1999. Characteristic analysis of a complex two-dimensional magnetohydrodynamic bow shock flow with steady compound shocks. Physics of plasmas, 6(3), 954-969.

[6] De Sterck, H., Low, B.C., \& Poedts, S. 2001. Overcompressive shocks and compound shocks in 2D and 3D magnetohydrodynamic flows. In: Proceedings of the Eighth International Conference on Hyperbolic Problems. International Series of Numerical Mathematics.

[7] Gibson, R.E., Schiffman, R.L., \& Cargill, K.W. 1981. The theory of one-dimensional consolidation of saturated clays. 2. Finite non-linear consolidation of thick homogeneous layers. Canadian geotechnical journal, 18, 280-293.

[8] KYnch, G.J. 1952. A theory of sedimentation. Transactions faraday society, 48, 166-176.

[9] Leveque, R.J. 1992. Numerical methods for conservation laws. Birkhäuser Verlag, Basel.

[10] Michaels, A.S., \& Bolger, J.C. 1962. Settling rates and sediment volumes of flocculated kaolin suspensions. Industrial and engineering chemistry fundamentals, 1(1), 24-33.

[11] OlEINIK, O.A. 1957. Discontinuous solutions of non-linear differential equations. American Mathematical Society Translations, 2(26), 95-172.

[12] Sills, G.C. 1998. Development of structure in sedimenting soils. Philosophical transactions of the Royal Society of London A, 356, 2515-2534. 